\title{
Experiences in Multi-domain Management Service Development
}

\author{
David Lewis and Thanassis Tiropanis \\ Department of Computer Science, University College London \\ dlewis@cs.ucl.ac.uk, ttiropan@cs.ucl.ac.uk \\ Lennart H. Bjerring \\ L.M. Ericsson A/S \\ lmdlhb@lmd.ericsson.se
}

\begin{abstract}
The developers of management systems and the management services that operate over them will be faced with increasing complexity as services are developed for the open service market. This paper presents experiences in the development of management services that span several administrative domains and which are therefore representative of the complexities of the open service market. The work described involved the development of TMN based management systems that provided management services in support of multimedia teleservices operating over broadband networks.
\end{abstract}

\section{$1 \quad$ Introduction}

As the structure of the telecommunications industry moves towards that of an open services market the role of telecommunication service developers will undergo a marked change. Developers will no longer be building services and management services for a single network but must utilise open interfaces to develop services over heterogeneous networks and platforms. Significantly, components that deliver these services will not just be under the administrative control of the service providers, but of the customer and the providers of other constituent services also. This requires, therefore, that the developers of services and, as addressed in this paper, of the accompanying management services must be aware of inter-domain issues.

This paper presents some of the experiences of the PREPARE project in developing inter-domain management services for deployment and demonstration on broadband testbed networks used for multimedia teleservices. This work was performed in two overlapping phases, the first started in January 1992 and resulted in a successful demonstration over a testbed based in Denmark in December 1994, the second started in January 1994 and will result in a demonstration in November 1995, operating on a testbed spanning three countries via the European PNO ATM Pilot [BBI94]. This paper will first outline some of the organisational and other non-functional requirements that characterised this work. It will then detail the experiences of the first phase, their influence on the second phase and the techniques that are therefore being used in this latter phase. Finally an assessment of our experiences and their relevance to future work will also be given. 


\section{Context}

The aim of the project was to gain experience in inter-domain management through the implementation of systems managing multimedia teleservices and the broadband testbed networks over which they operate. The project's consortium of collaborating partners contained representatives of many of the players in an open service market, including public network operators, public network equipment vendors, customer premises equipment vendors, multimedia teleservice developers and management platform vendors. The consortium structure, however, introduced an artificial flavour to the process of developing the management services by effecting the way the design and implementation work was distributed between different organisations. The pattern of a provider analysing and developing a service for commercial customers was not followed, but instead, as in any collaborative research project, the work had to accommodate the aspirations held by and restrictions imposed on each of the partners involved.

In general the development of a methodology for system design, whether the system is an inter-domain management system or something else, can be seen as an application of the overall principle of "separation of concerns", i.e. the partitioning of a complex problem space into a number of smaller, more manageable problem spaces. A useful methodology for a project like PREPARE is therefore deeply dependent on the nature of the system to be designed, and at least as dependent on the background knowledge and experience of the people who are to use the methodology. What we do hope to provide here is therefore an illumination of the complexity of the overall task of designing inter-domain management systems by identifying the aspects of the task which in our experience are the most relevant and important ones. The necessary background knowledge of people is in-depth knowledge about TMN [TMN] and OSI-SM [X700] architectural principles and communications mechanisms.

A further influence on this development work was the relative immaturity of standards dealing with inter-domain management. The ITU-T's TMN development methodology [M3020] did not at the start of the project fully address the issues involved in developing service level inter-domain management systems but rather concentrated on the development of network and network element level information models. The ODP was also considered [ODP]. It was seen initially as being too broad to apply to the specific inter-domain interface development work, but has been referred to more in the later, more complicated phases of the project, though not in a rigorous manner. The use of NMF Ensembles [Ensemble] was also considered in the second phase. It was regarded as providing an excellent way of expressing the requirements and designs and has been adopted for their documentation. The lack from the start of PREPARE of existing Ensemble definitions relating to inter-domain management has however precluded a development path closely integrated with the OMNIPoint strategy, since we have, from the start of the first phase, had to produce our own base specifications (e.g., information models) which then were reused and further enhanced in the second phase. 


\section{$3 \quad$ Experiences from the first testbed}

\subsection{Requirements and limitations}

The main focus of the first development phase was on the end-to-end management of resources of diverse networks owned and administered by different organisational domains. This was reflected in the first phase testbed which was made up of ATM and Token-Ring LANs, a DQDB MAN and an ATM WAN [BBI93]. The PREPARE team at that time had experience in network resources management and deployment of management systems. The biggest challenge was to demonstrate end-to-end management of resources over heterogeneous equipment and management software. This required a effective, well-defined methodology and, at the same time, an awareness of the practical limitations.

One area where practical limitations combined with individual partner requirements in shaping the approach taken was that of management platforms. TMN recommendations and OSI management were chosen as the basic technology and different platforms were available to different partners, namely OSIMIS, HP OpenView, IBM's Netview/6000 and later TMN/6000 and Ericsson's TMOS. However the current state of OSI management platform technology does not provide for a standard high level API. The industry standard XMP/XOM was found to be too low level for the volume of development required and in some cases was augmented with a higher level interface. A high level API had been developed for manager applications which provided managed object location transparency by providing a single interface to X.500 and X.700 objects [CFS H430], but this was not ported to all platforms in time to support the project's development cycle as a common API. This lack of a common programming API therefore led to a situation where individual TMN operations systems (OS) were developed by different partners on different platforms, precluding the development of common service software modules that could operate on several platforms. This in turn led, in the initial stages of the project at least, to a concentration of effort on the careful definition of OS functionality as governed by its behaviour at inter-domain interfaces (X-interfaces in M.3010). As will be explained below, as experience of inter-domain interface definition grew, the emphasis in the second phase changed towards specifying the broader management functionality of co-operating systems also.

\subsection{Approach}

The approach to be taken for the design and implementation of the PREPARE interdomain management system was not clear at the beginning of the project. The standardised methodologies discussed above did not seem to provide an approach that integrates the service specification, design and implementation phases of interdomain management services.

The first stage of the initial plan was the definition of management scenarios to be demonstrated, the definition of management services and the definition of initial information models for inter-domain management. After the completion of this stage, 
the next step was the implementation of the intra-domain systems for the subnetworks and then the implementation of the inter-domain components.

For the first stage, four groups were formed:

- Scenarios group. Its aim was to produce a set of management scenarios to reflect a representative subset of management functionality to be demonstrated, set in a realistic enterprise context.

- Architecture group. It focused on specifying an implementable TMN based framework for interfacing the components in each domain.

- Management Services group. Its work was to define a set of services to operate between the OSs of the different domains.

- Information Modelling group. It worked on the definition of information models required for the different OSs involved in inter-domain management according to the GDMO recommendations [GDMO].

For the second stage the output these groups produced had to be refined to a form that could be implementable. The scenarios were refined to describe the flow of information identified in the initial information model between the management OSs identified in the TMN architecture. These information flows then provided the basis for refinement of the information models for each OS.

When the information model became stable, the team produced Test Design Specifications (TDS) according to the IEEE standard 829-1983. The TDSs described all the tests that involved components from two or more partners. The test cases derived from the scenarios produced by the scenarios group after the refinement through the information flows and the information modelling. The final level of detail involved defining the CMIP operations between the OSs and all the parameters of the information to be exchanged. The fact that all this detail was specified before the implementation proceeded too far was of great importance, because it gave the opportunity to address and resolve many technical issues that had not been considered during the early stages of the design.

The main stages of the methodology followed by PREPARE in its first stage can therefore be summarised as:

- Definition of scenarios to clarify the aims of the whole development and the relationships between the different actors on the enterprise level.

- Information flows and Information Models to outline the interactions that take place between the OSs to provide end-to-end management as well as the information structuring of the components in each OSs for inter-domain management.

- Test Design Specifications which describe in detail the lower level management interactions between OSs as well as the information to be exchanged between them during the execution of the scenarios. 


\section{$4 \quad$ Experiences from the second testbed}

The second phase of the project was intended to build upon the first phase both in terms of experiences gained in the inter-domain management service design process and in the extension of the physical testbed and the specific services that operated over it [ISN94].

\subsection{Requirements and Limitations}

The second phase differed from the first in that the enterprise situation was more complicated, involving more service providers and relationships between service providers and the range of scenarios being addressed was to be more ambitious. There was also a change in emphasis from simply producing service level OSs in each to domain, to adding WSFs with rich functionality for service and network administrators in each domains. It was however similar to the first phase in that the physical management architecture had a TMN-based structure and that the effect of implementing on multiple management platforms was still in evidence.

The actual enterprise situation involved separate multimedia conferencing teleservice and multimedia mail global store teleservice providers providing their services to users on remote customer premises networks (CPN). The teleservice providers used the services of a separate virtual private network (VPN) provider to manage end-toend network resources over several public network domains and the CPN domains to support the teleservices' communication needs.

\subsection{Approach}

Based largely on the experiences of the first phase, it was felt that a more cohesive working approach was required. All the analysis, specification, design and implementation was therefore performed in one homogenous group which would split into subgroups at various stages to address clearly defined functional areas rather than splitting into groups addressing the different stages themselves. The approach taken again had to combine both top down and bottom up aspects, though in this case more attention was paid to some of the top down design aspects due to the increased complexity of the enterprise situation. The work can be broken down into the following areas:

- Enterprise Modelling and Scenario Description: this laid out the organisational context in which the work of this phase would be and defined, in the form of scenarios, the scope of the management functionality to be addressed within this context.

- Management Architecture Design: this provided the physical architecture of the TMN systems that would provide the framework for the more detailed design work.

- Role Specifications: These provided a way of describing in more detail the requirements of the involved organisations through the definition of 
responsibilities for individuals identified in the enterprise model and a way of mapping these requirements to lower level management function requirements.

- Information Modelling and Information Flows: This involved the identification of information required to perform the required management functions, their definition as information models representing each domain and the definition of inter-domain operations performed on this information model in support of the required management functions.

- Operations System Design: This involved the functional design of the various TMN operation systems. These were identified in the management architecture in accordance with the requirements defined by the role specifications as specified by the inter-domain interfaces, which in turn are defined by the information models and information flows.

- Workstation Function Design: This area addressed the particular requirements of the user interface for the individuals operating the management services. It was therefore closely related to both the Role Specifications and the Management Function Design.

It should be made clear that these various areas were not addressed sequentially but were to an extent interleaved with some being revisited after the initial work on others had provided clearer insight into the requirement upon them. Each of these areas are described in more detail in the following sections.

\section{Enterprise Modelling and Scenarios}

This area identifies the organisational stake-holders, their characteristics (e.g. core business area) and focus on the objectives for their involvement in order to identify their main high-level requirements on the system and each other. Based on these organisational structures are considered and lead to the identification of roles. We concentrated on inter-domain aspects, i.e. inter-organisational relationships where agents of the stake-holder organisations interact on behalf of their organisations. The overall domain structure was also considered here, and motivates our use of the term inter-domain management.

\section{Role Specifications}

In an inter-domain service management system it is very important to distinguish between the roles in the various domains and to establish the relationships between these roles as it is here that inter-domain management functionality is required that must be supported by the inter-domain service management infrastructure. Role specifications were therefore adopted as a means to ensure that the management functionality required by the role holders in the scenarios was adequately described and could be supported by the inter-domain management system infrastructure that was being implemented.

The specific roles required for the demonstrator were first identified via the scenario descriptions. A common role specification template was adopted as a means to structure in a similar manner for all roles what the actual role entails, and by 
refinement to be able to map from the role specification down to the operations on the resources, and finally as a result of information modelling to the managed objects.

This template was developed based on the work of the ESPRIT project ORDIT, which investigated the organisational requirements for information technology systems by examining roles and responsibilities within an organisation [ORDIT]. In PREPARE the ORDIT concepts were adopted for the specific needs of the role specification work and the particular aims of PREPARE regarding inter-domain service management for the second testbed. Therefore the role specification work was concerned with specifying the responsibilities of the various roles and the relationships between them, according to their depiction in the scenarios. On the basis of the responsibilities, obligations were described, and based on these, the activities associated with each obligation could be described. The role specification template therefore included for each role holder the responsibilities of the role holder and to whom, the obligations that need to be discharged by the role holder in order to meet the responsibilities of the role, the activities which need to be carried out to enable the role holder to fulfil the obligations deriving from the responsibilities and the resources and access rights required to enable the role holder to carry out the activity.

\section{Management Architecture Design}

The management architecture referred to here is the physical architecture of the total inter-domain management system. The architecture is designed, in common with that of the first phase, based on the following principles: each organisational stakeholder has its own associated TMN; organisations which own physical network technology has within their TMN an operations system (OS) which implements management functions (OSFs) specific to the particular network being managed by that TMN, a Network Operations System (N_OS); each TMN has a Service Operations System (S_OS) implementing service management functions associated with that particular domain, and which takes part in the overall distributed end-to-end service management function execution. This means that N_OSs inter-operate with the S_OSs in their own TMN (via Q3-type interfaces) and that S_OSs inter-operate with other S_OSs in other TMNs (via X-type interfaces). Management end-users (e.g. CPN administrators, VPN administrators) access the pertinent management function by TMN Workstations interfacing the pertinent operations system. The figure 1 presents an overview of the resulting physical management architecture.

Attention was paid in this area to explicitly addressing the non-functional requirements imposed by the scope of partners interests, the platforms available to partners and to reducing the overall complexity of the information modelling and information flow definition tasks by minimising the number of inter-domain interfaces involved. Significantly by addressing these issues at the architectural stage of the design process it was found that it was subsequently easier to split the work between relatively independent groups addressing functionality in different areas of the physical architecture. 


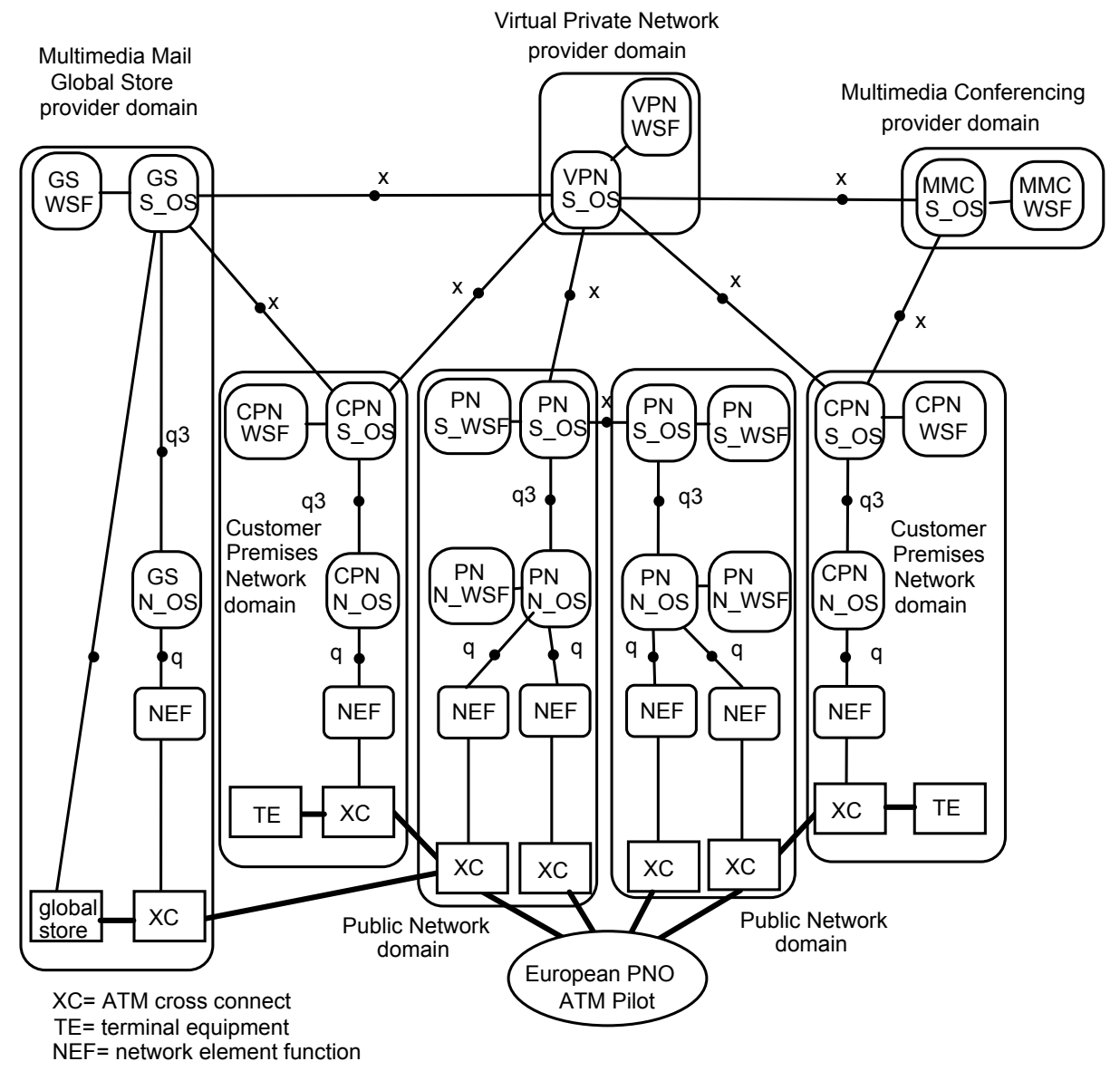

Figure 1: Second Phase TMN Architecture

\section{Information Models and Information Flows}

Initial information models based on the requirements imposed by the enterprise model and the scenario descriptions were made more concrete by the role specification process. Following the pattern well established in the first phase, this information model was mapped onto the physical architecture. Information flows were then generated, detailing how the management activities outlined in the scenarios are accomplished by operations on managed objects. An information flow describes a management function implementation in terms of CMIS message flows between two management applications, thus it describes which MOs are being managed, which operations are sent to them, and which attributes and parameters are transferred between the two management applications. Information models and flows need to be designed in an iterative fashion, since information flows will identify missing 
information which needs to be included in the information model specification and verified through updated information flows.

\section{Operations System Design}

The functionality of the OSs identified in the TMN architecture was governed generally by the requirements of the scenarios and role specification with their interfaces to other OSs and the functional interactions between different interfaces defined in the information models and the information flows. Once this level had been achieved however the functional design of individual OSs was left largely to the judgement of individual designers.

In a few cases, however, where the development of several OSs with common functional requirements was shared between two partners a more formal approach was taken to the functional design of the OSs. This work took an object oriented approach loosely based on some of the output of the TINA Consortium [TINA]. This involved defining functional building blocks that addressed specific functional areas, e.g. billing or the management functionality required by the customer of a specific service, and could be used in different OSs as required by the enterprise model. These building blocks were then further decomposed into computational objects that provided both the functional structure of the building blocks but also the interfaces offered by this functional building block to other functional building blocks. The computational objects were defined with multiple interfaces to explicitly differentiate between the functions and access rights required by the different roles identified in the role specifications. The final design then consisted of mapping these computational objects onto engineering objects that implemented the OS functionality.

\section{Workstation Function Design}

Workstation Functions (WSF) provide the representation of systems and sub-systems as relevant and needed by a role holder, taking various concerns into account. The WSFs design depends to a large extent on platform technologies, in that such platforms often have individual style guides prescribing many aspects of the GUI, for instance use of colours and maps, window layout and menus. The role specifications however provided important indications of what was to be represented on the screen (the resources the role holder manages or is aware of), and the capabilities over these resources which are available to the role holder (which for instance would provide clear indications for the contents of menus associated with each resource).

\section{$5 \quad$ Assessment and Further Work}

The PREPARE project did not have as one of its objectives the development of an explicit methodology for developing inter-domain management systems and the work presented here should therefore not be assessed on this basis. However this work has provided, we believe, some useful insights into and experiences of the processes involved in such development. Some of these insights are implicit in the evolution from the first to the second phase described above, but others are assessed in more 
detail below, together with areas of further work the project consortium aims to follow in this area.

The ORDIT based role specification aspect of the second phase allows a top-down method that is intended to ensure that all the functionality needed to be implemented in the scenarios is covered. As a top-down approach, the role specification process allows the services and their users to be perceived in a service environment rather than in a network environment. The responsibilities, obligations, and activities that are defined exist regardless of the specific underlying technology, and by focusing on the service a more long-term, service-oriented view is determined, i.e., the emphasis is on what the service offers rather than how it is implemented. Especially in an open service market it is important to consider services from the user and customer viewpoint and the role specification approach supports this by examining the requirements that users have on the management system in order to carry out their tasks. Therefore role specifications provide a suitable methodological approach to capture this very important customer and user-oriented aspect of service management. Role specifications can be seen as a means to define and analyse the main business objectives, interests and motivations for various stake-holders' involvement. This enables design of solutions which fit into the application context of the customers and users. Therefore, domain/stake-holder analysis, followed by work role analysis was found very useful.

The area of functional architecture and specification has not been addressed fully in the project. Issues concerning the definition of functional components for interdomain service management must be dealt with in more detail if functional components of different services are to be integrated to manage value added services and if functional component reuse is to be encouraged. This requires the specification of a functional architecture to complement the physical TMN architecture and information models.

The PREPARE consortium plans to continue working together in the ACTS programme to investigate the application of inter-domain management to new services and will in the process continue to examine and refine the methodologies used to develop working systems. In the time frame of the ACTS programme work from standards bodies and industrial consortium will provide more relevant input into PREPARE (some of it influenced by PREPARE initially). The NMF is investigating issues of service management [NMF] and some of PREPARE's results in this area expressed in Ensemble format are being prepared as input to this group.

The TINA Consortium is also promising some relevant results that could be applied to multi-domain service design. Its highly object oriented approach to defining service components containing integrated management services as utilised for some OS functional design, may prove useful in the future. Currently however TINA's reliance on a Distributed Processing Environment providing object location transparency, tends to obscure some issues of inter-domain information modelling related to ownership of resources, protection of data from failure in other domains and associated security. There is nothing, however, preventing these issues being 
addressed as specific services in the TINA-C architecture, and this would be a likely area of research for the PREPARE consortium.

\section{Conclusions}

The PREPARE project is now in its fourth year of designing and implementing TMN based management systems operating inter-domain management services. This period has seen an increase in the experience of techniques required to perform this task successfully together with a greater understanding of the specific problems invoked by the inter-domain nature of this area. Techniques of iteratively refined information and scenario-based information flows have proven adequate for designing simpler systems, but more complex enterprise situations and wider ranges of management activities need more attention to top down techniques. In particular, where multiple stake-holders are involved in a service, the use of role specifications provides a suitable method for mapping the overall management service functionality to individual role activities and OS functions. The application of TMN principles still largely rotates around the definition of interfaces between components in the physical architecture however. The efficient use of management services in an open service market will require more emphasis on the definition of functional architectures and components.

\section{Acknowledgement}

This work was partially funded by the commission of the European union under the RACE II program, project number 2004. The view presented in this paper are not necessarily those of the PREPARE consortium.

\section{References}

[TMN] ITU-T Recommendation M.3010 (1992), Principles for a TMN.

[X700] ITU-T Recommendation X.700, OSI Systems Management.

[ISINM] D.Lewis, W. Donnelly, S. O'Connell, L.H. Bjerring, "Experiences in Multi-domain Management System Development", Proceedings of the International Symposium on Integrated Network Management, Santa Barbara, 1995

[ORDIT] R. Strens, J. Dobson, "Responsibility Modelling as a Technique for Organisational Requirements Definition", Intelligent Systems Engineering, 3 (1), 1994, pp.20-26

[ISN94] L.H.Bjerring, J.M.Schneider, "End-to-end Service Management with Multiple Providers", Proceedings of the IS\&N Conference, Aachen, 1994

[M3020] ITU-T Recommendation M.3020, "TMN Interface Specification Methodology" 
[TINA] H. Berndt, L. de la Fuente, P. Graubmann, "Service and Management Architecture in TINA-C", Proceedings of TINA Conference, Melbourne, 1995

[NMF] Network Management Forum, "A Service Management Business Process Model" , 1995

[BBI94] D. Lewis, P. Kirstein, "A Broadband Testbed for the Investigation of Multimedia Services and Teleservice Management", Proceedings of the 3rd International Conference on Broadband Islands, Hamburg, 1994

[BBI93] D. Lewis, P. Kirstein, "Multimedia Applications and Services in the PREPARE Testbed", Proceeding of the 2nd International Conference on Broadband Islands, Athens, 1993

[ODP] ITU_T, Recommendation X.900 Series, "Basic Reference Model for Open Distributed Processing", ISO 10476

[Ensemble] Network management Forum, "The Ensemble Concepts and Format", Forum 025, Issue 1.0, August 1992

[GDMO] ITU-T Recommendation X.722, "Guidelines for Definition of Managed Objects"

[CFS H430] RACE CFS H430, "The Inter-Domain Management Information Service (IDMIS)". Issue D, 1994 